\title{
Enhancing the Coherent Detection of Optical QAM Signals by Exploiting the Information of Received Optical Power
}

\author{
Shenli Zhang ${ }^{\ddagger}$, Bin Chen ${ }^{\ddagger}$, Linghao Cheng*, Zhaohui Li*, Chao Lu , and Hui Wang ${ }^{\ddagger}$ \\ ${ }^{\ddagger}$ Communication Engineering Departmetn, college of information engineering, Shenzhen University, China. \\ (e-mail: zsl@szu.edu.cn, bchen@szu.edu.cn,wanghsz@szu.edu.cn). \\ * Institute of Photonics Technology, Jinan University, China (e-mail: chenglh@ieee.org, tlzh88@jnu.edu.cn) \\ dept. of electronic \& information engineering, Hong Kong polytechnic unviersity, Hongkong (e-mail: Chao.Lu@inet.polyu.edu.hk)
}

\begin{abstract}
In current optical coherent communication systems, balanced photo-detectors are typically employed, which preserve the product of the received optical signals and the local oscillators (LO) for detection but cancel out the power of both. Since only the product of the received optical signals and the $L O$ is used to estimate the transmitted complex symbol, such detection scheme is suboptimal due to the loss of some useful information embedded in the transmitted signal power. Different from that, this paper proposes a new detection scheme which maintains full information obtained from the photo-detectors. Specifically, we first extract the transmitted signal power from the output of the photo-detector. After that, we use not only the product of the signals and the LO but also the power of signals to estimate the target signals. For 16-qudrature-amplitudemodulation (16-QAM) and 64-QAM signals, the simulation results show that our new scheme can outperform the conventional ones by more than $1 \mathrm{~dB}$.
\end{abstract}

Index Terms - Coherent detection, optical fiber communication, quadrature-amplitude-modulation (QAM).

\section{INTRODUCTION}

With rapid growing demand on capacity, there has been renewed interest in coherent detection for optical fiber communication systems [1]. Coherent optical transmission can preserve all the information of optical fields during detection, enabling possibilities for optical multi-level modulations [2]. Many works have been done on quadrature-phase-shift-keying

This project was supported by the National Science Foundation of China (NSFC) projects with No. 60902016, 60773203. It was also supported by the science foundation of Shenzhen University with No. 200865.
(QPSK), 8PSK and quadrature-amplitude-modulation (QAM) to get spectral efficiency of more than $2 \mathrm{bit} / \mathrm{s} / \mathrm{Hz}[3-5]$. Higher spectral efficiency results in lower symbol rate for a given transmission rate (in bit), which brings many advantages such as higher tolerance to chromatic dispersion (CD) and polarization mode dispersion (PMD), lower requirement on the bandwidth and speed of analog-to-digital converter (ADC) and of digital signal processing (DSP). However, on the other hand, modulations with higher spectral efficiency normally show poorer sensitivity [6]. As the launched optical signal power is ultimately limited by fiber nonlinearity for optical fiber communication systems, the link reach can not simply be extended by increasing the transmitted signal power. Therefore, it is quite critical to explore other methods to improve the sensitivity.

For BPSK, QPSK or M-PSK optical transmission systems, the symbol values are decided by the phase of the transmitted signal while the amplitude of the transmitted signal is fixed. Hence the product of the transmitted signal and the local oscillator, as widely used in traditional optical receiver, is enough for signal detection and decision. For more than 8 phases PSK modulation, however, the error-rate is too high to be feasible for real applications. As the spectral efficiency further increases, coherent QAM becomes a very attractive way [5]. However, different from the constant amplitude in M-PSK systems, the constellation points have not only different phases but also different amplitudes in QAM optical transmission systems. In this case, both the amplitude information and the phase information are useful. However, current optical QAM coherent detecting process still only uses the product of the transmitted signal and the local oscillator, ignoring the amplitude information which is actually provided by the photo-detectors. As a result, there is a potential loss of some useful information in current detectors for QAM symbol detection. 
In this work, we propose a new detection scheme that fully exploits the information of the signal power to estimate the signal amplitude and improve the overall system performance for optical QAM signals.

This paper is organized as follows. In section II, the system model and the conventional coherent detection scheme in optical systems are introduced. In section III, our new detection scheme is detailed and it's performance is simulated in section IV. Finally, section V concludes our paper.

\section{SySTEM MODEL AND CONVENTIONAL COHERENT DETECTION SCHEME}

The basic idea of coherent detection is a phase-diversity receiver as shown in Fig. 1. To detect the in-phase signal, the transmitted optical signal $E_{S}$ and the photonic local oscillator (LO) $E_{L O}$ are coupled through an optical coupler which produces two outputs with one for the sum and the other for the difference of $E_{S}$ and $E_{L O}$. The two outputs are then detected by photo-detectors. The detection of the quadrature-phase signal is the same as that for the in-phase signal except that $E_{L O}$ experiences a $\pi / 2$ phase shift. The photo-detectors employed in the phase-diversity receiver are essentially square-law detectors [7]. Therefore, the two in-phase signals and the two quadrature-phase signals after photo-detectors can be expressed as:

$$
\begin{aligned}
& I_{I 1}(t)=\frac{R}{2}\left(P_{S}+P_{L}+2 \sqrt{P_{S} P_{L}} \cos \left(\theta_{s}(t)+\theta_{n}(t)\right)\right)+n_{1} \\
& I_{I 2}(t)=\frac{R}{2}\left(P_{S}+P_{L}-2 \sqrt{P_{S} P_{L}} \cos \left(\theta_{s}(t)+\theta_{n}(t)\right)\right)+n_{2} \\
& I_{Q 1}(t)=\frac{R}{2}\left(P_{S}+P_{L}+2 \sqrt{P_{S} P_{L}} \sin \left(\theta_{s}(t)+\theta_{n}(t)\right)\right)+n_{3} \\
& I_{Q 2}(t)=\frac{R}{2}\left(P_{S}+P_{L}-2 \sqrt{P_{S} P_{L}} \sin \left(\theta_{s}(t)+\theta_{n}(t)\right)\right)+n_{4}
\end{aligned}
$$

where $R$ is responsitivity of the photo-detector [7]. Since the local signal power $P_{L}$ is a local parameter and it's value is known to the receiver, we simply assume it to be unit for simplicity. $P_{\mathrm{s}}$ is the power of the transmitted signal. $\theta_{s}(t)$ is the phase of the transmitted signal. $\theta_{n}(t)$ is the phase offset, which is assumed to be perfectly known[1]. For simplicity, we assume the noise $n_{i}$ is of Gaussian distribution $N\left(0, \sigma^{2}\right)$ and we further assume that $n_{i}$ is independent of $n_{k}$ if $i \neq k$.

For the balanced detection scheme employed in most of current coherent detection systems, the estimate of the transmitted in-phase signal is only based on the product of the transmitted signal and the LO signal as shown below

$$
\begin{aligned}
I_{I}(t) & =\left(I_{I 1}(t)-I_{I 2}(t)\right) /(2 R) \\
& =\sqrt{P_{S}} \cos \theta_{s}(t)+\left(n_{1}-n_{2}\right) /(2 R)
\end{aligned}
$$

In the similar way, the estimate of the transmitted quadraturephase signal is

$$
\begin{aligned}
& I_{Q}(t)=\left(I_{Q 1}(t)-I_{Q 2}(t)\right) /(2 R) \\
& \quad=\sqrt{P_{S}} \sin \theta_{s}(t)+\left(n_{3}-n_{4}\right) /(2 R)
\end{aligned}
$$

By quantizing the two signals in (2) and (3), the hard-decision of the transmitted complex symbol, $\sqrt{P_{S}} e^{j \theta_{S}(t)}$, can be obtained.

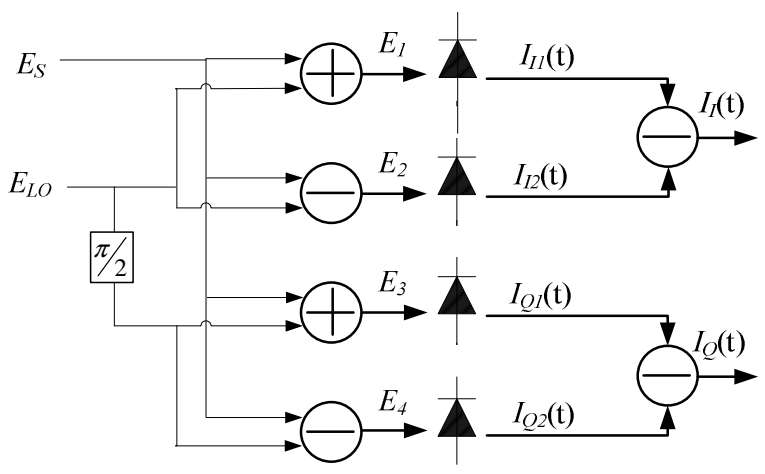

Fig. 1. Conventional phase-diversity receiver employed in current optical transmission systems for coherent detection.

\section{Proposed COHERENT DeteCtion SCHEME}

Although the conventional detection method works in practical systems [7], the operations shown by Eq. (2) and Eq. (3) lose some information that may help the detection processing. The photo-detectors actually provide information on the optical power of the transmitted signal and the LO signal as shown in Eq. (1), but this information is discarded by the balanced detections as shown by Eq. (2) and Eq. (3). For QAM signal, the power of the transmitted signal also contains valuable information to enhance the detection performance. We here propose a novel detection scheme as shown in Fig. 2, which tries to use all the useful information from the photo-detectors. 
Besides the two outputs obtained from the conventional detection scheme, we can sum up all the outputs of the four photo-detectors to get a new output denoted by $P_{A}$, which represents the received optical power. In particular, besides $I_{I}$ in Eq. (2), we can also obtain an estimate of the transmitted signal power as

$$
y_{1}=\left(I_{I 1}(t)+I_{I 2}(t)\right) / R-1=P_{S}+\left(n_{1}+n_{2}\right) / R
$$

As the linear transformation from $\left(I_{I 1}, I_{I 2}\right)$ to $\left(y_{1}, I_{I}\right)$ is invertible [8], $y_{1}$ and $I_{I}$ contains the same amount of information about the target signal $\sqrt{P_{S}} e^{j \theta_{S}(t)}$ as $I_{I 1}$ and $I_{I 2}$,

$$
I\left(\sqrt{P_{S}} e^{j \theta_{S}(t)} ; y_{I_{1}}, y_{I_{2}}\right)=I\left(\sqrt{P_{S}} e^{j \theta_{S}(t)} ; I_{I}, y_{1}\right)
$$

where $I(x ; y)$ is the mutual information between $x$ and $y$. In other words, there is no information loss for the transformation from $\left(I_{I 1}, I_{I 2}\right)$ to $\left(y_{1}, I_{I}\right)$. Based on similar argument, $I_{I}$ only contains partial information about the target signal. From the quadrature-phase signals, we will obtain another estimate of the transmitted signal power as

$$
y_{2}=P_{S}+\left(n_{3}+n_{4}\right) / R
$$

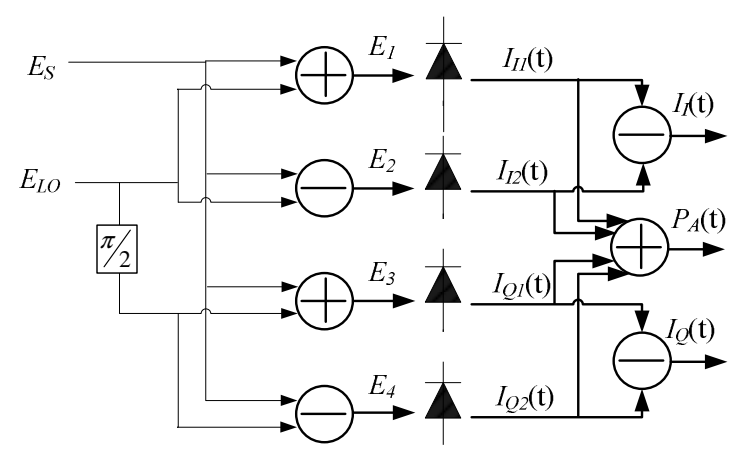

Fig. 2 Proposed phase-diversity receiver with additional output $P_{A}$ representing the received optical power to enhance the coherent detection.

We now have four variables, $I_{I}, I_{Q}, y_{1}$ and $y_{2}$, which contain all the information obtained by the photo-detectors. We can use them to estimate the in-phase and quadrature-phase components of the target signal. To do so, we first estimate the amplitude of the target signal. Combining $y_{1}$ and $y_{2}$ with equal gain, we can obtain one estimate of the amplitude as

$$
\begin{aligned}
& x_{1}=\sqrt{\left(y_{1}+y_{2}\right) / 2}=\sqrt{P_{A} / R-2} \\
& =\sqrt{P_{S}+\left(n_{1}+n_{2}+n_{3}+n_{4}\right) /(2 R)}
\end{aligned} .
$$

We can also obtain one estimate of the amplitude from $I_{I}$ and $I_{Q}$. The phase can be estimated as

$$
\tilde{\theta}_{S}=\operatorname{Angle}\left(I_{I}+j I_{Q}\right)
$$

where the function Angle( ) gives the angle of one complex value. Then, we can estimate the signal amplitude as the following ${ }^{1}$

$$
x_{2}=\left(I_{I} / \cos \left(\tilde{\theta}_{S}\right)+I_{Q} / \sin \left(\tilde{\theta}_{S}\right)\right) / 2
$$

Generally speaking, the noise components in Eq. (8) and Eq. (9) are independent. Therefore, we can combine the two estimates to obtain a better estimation of the signal's amplitude as

$$
\sqrt{\tilde{P}_{S}}=\left(\alpha x_{1}+\beta x_{2}\right) /(\alpha+\beta)
$$

where $\alpha$ and $\beta$ are combination coefficients.

Finally, we quantize the in-phase part $\sqrt{\tilde{P}_{S}} \cos \left(\tilde{\theta}_{S}\right)$ and the quadrature-phase part $\sqrt{\tilde{P}_{S}} \sin \left(\tilde{\theta}_{S}\right)$ to obtain the hard decision.

Remark: If we can calculate the signal-to-noise-ratio (SNR) in Eq. (8) and Eq. (9), the theoretical optimal combination coefficients $\alpha$ and $\beta$ can be obtained by the maximum ratio combination [9]. However, due to the non-linear processing in Eq. (8) and Eq. (9), it is difficult to calculate the theoretical SNRs. As in the next section, we can use the simulation method to find the optimal combination coefficients. In fact, we found that the SNR of $x_{1}$ is much larger than the SNR of $x_{2}$, and it is good enough to only use $x_{1}$ as the estimate of target signal's amplitude.

\section{Simulation RESUlts}

In this section, numerical simulation is done to evaluate the performance of our proposed detection scheme. For simplicity, we fix $R$ to 2 . The signal's power $P_{S}$ equals $4 E_{b}$ for 16-QAM and it equals $8 E_{b}$ for 64-QAM, where $E_{b}$ is the transmitting energy on each bit. In our simulation, we first randomly generate the source bits and modulated them with 16QAM or

${ }^{1}$ In fact, the amplitude can also be estimated as $\sqrt{I_{I}{ }^{2}+I_{Q}{ }^{2}}$. Our simulation shows that this estimate performs almost the same as that in (9). Therefore, we ignore the discussion of this method. 
64QAM. Then we can obtain the power of the transmitted symbol, the product between the transmitted symbol and the local symbol and power of the local signal as in Eq. (1). By adding randomly generated Gaussian noise, the received form symbol is obtained. After that, we demodulate the received symbol according to the traditional scheme (Eq. (2) and Eq. (3)) and the proposed scheme (Eq. (10)), respectively. At last, the symbol error rate is calculated.

In Fig. 3, the symbol error rate (SER) for 16-QAM is plotted. When $\alpha=0$ and $\beta=1$, i.e., only $x_{2}$ is used as the amplitude, the SER performance of the proposed scheme is almost the same as the conventional scheme since the information in $y_{1}, y_{2}$ is not exploited. When $\alpha=1$ and $\beta=0$, i.e., only $x_{1}$ is used as the amplitude, the SER performance of the proposed scheme outperforms the conventional scheme by about $1 \mathrm{~dB}$ (at a SER of about 1E-2), which means that the distortion of the amplitude in $x_{1}$ is much smaller than that in $x_{2}$. When $\alpha=16$ and $\beta=1$ which are combination coefficients obtained by offline simulation, the SER performance is slightly better than the case that $\alpha=1$ and $\beta=0$. On one hand, this fact means that the noise components in $x_{1}$ and $x_{2}$ are really independent. On the other hand, it means that the amplitude distortion in $x_{1}$ is much smaller than that in $x_{2}$ and it is good enough to ignore the amplitude information in $x_{2}$.

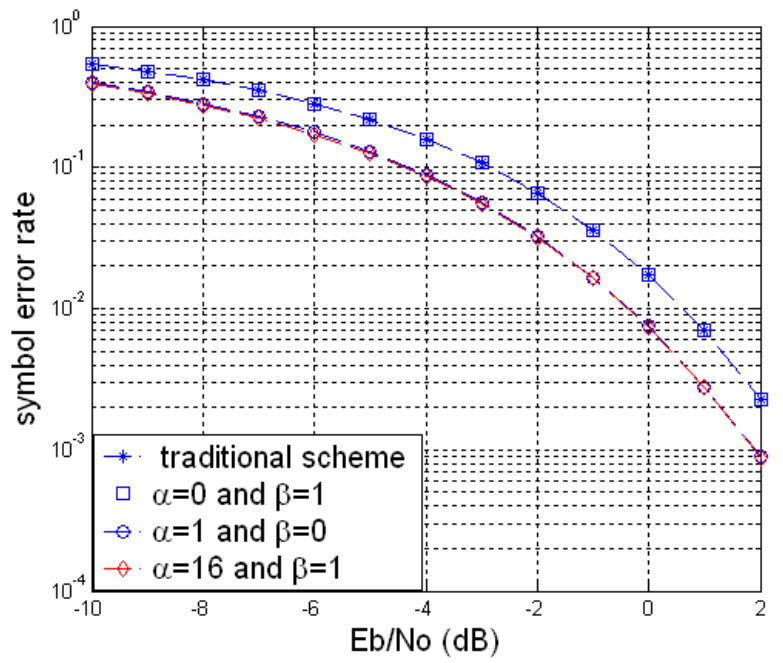

Fig. 3, 16-QAM SER performance of different detection schemes

In Fig. 4, the SER for 64-QAM modulation is plotted based on the same simulation setting. We can find the same performance relations as in Fig. 3 except that our new schemes can achieve more performance improvement (about $1.5 \mathrm{~dB}$ at a
SER of 1E-2). The reason is that there are more amplitude levels in 64QAM than in 16QAM. Therefore, to accurately estimate the amplitude becomes more important.

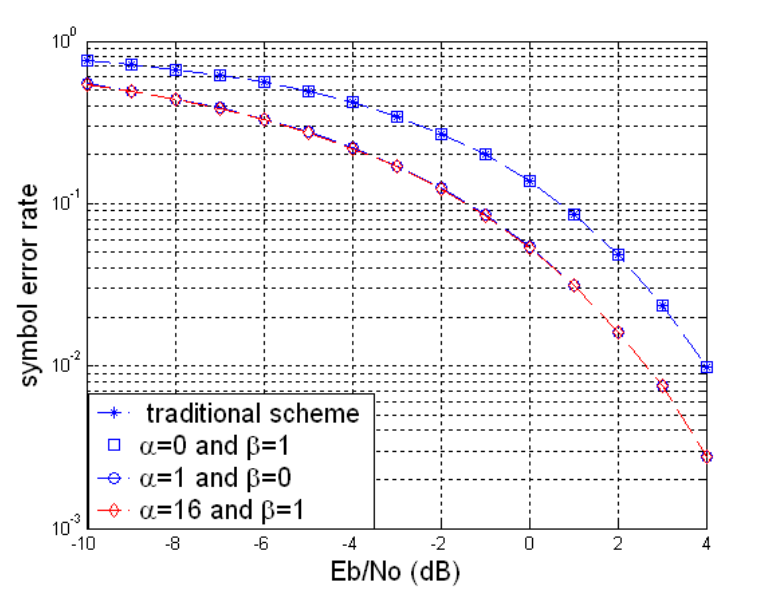

Fig. 4, 64-QAM SER performance of different detection schemes

\section{CONCLUSION}

In this work, we try to exploit the full information obtained from the photo-detectors to improve the signal estimation in coherent optical fiber communication systems. The results show that our method can give more than $1 \mathrm{~dB}$ SNR improvement for 16QAM optical signals and $1.5 \mathrm{~dB}$ for 64-QAM optical signals. Higher order optical QAM signals are very likely to benefit more by using our method.

\section{REFERENCES}

[1] K. Kikuchi, "Coherent optical communications -history, state-of-the-art technologies, and challenges for the future-," in proc. of OECC/ACOFT, Jul. 2008.

[2] X. Zhou, and J. Yu, "Multi-level, multi-dimensional coding for high-speed and high-spectral-efficiency optical transmission", J. of Lightwave Technol., vol. 27, no. 16, pp. 3641 - 3653, Aug. 2009.

[3] K. Roberts, M. O'Sullivan, K.-T. Wu, H. Sun, A. Awadalla, D. J. Krause, and C. Laperle, "Performance of dual-polarization QPSK for optical transport systems", J. of Lightwave Technol., vol. 27, no. 16, pp. 3546 3559, Aug. 2009.

[4] X. Zhou, J. Yu, D. Qian, T. Wang, G. Zhang, and P. D. Magill, "High-spectral-efficiency 114-Gb/s transmission using polmux-RZ-8PSK modulation format and single-ended digital coherent detection technique", J. of Lightwave Technol., vol. 27, no. 3, pp. 146 152, Feb. 2009

[5] M. Nakazawa, "Optical quadrature amplitude modulation (QAM) with coherent detection up to 128 states", in proc. of OFC/NFOEC 2009, Paper OThG1.

[6] A. H. Gnauck and P. J. Winzer, "Optical phase-shift-keyed transmission," J. Lightw. Technol., vol. 23, no. 1, pp. 115-130, Jan. 2005.

[7] I. P. Kaminow, T. Li, Alan E. Willner, "Optical Fiber Telecommunications V B: Systems and Networks 5th Edition Kaminow, Ivan P.” Publisher Elsevier, 2008, ISBN: 978-0-12-374172-1. 
[8] T. M. Cover, J. A. Thomas, "Elements of Information Theory", Wiley-Interscience, (August 12, 1991).

[9] J.G. Proakis, Digital communications ( $4^{\text {th }}$ ed.), New York: McGraw-Hill, 2003. 